\title{
ELLIPTIC EQUATIONS OF HIGHER STOCHASTIC ORDER
}

\author{
Sergey V. Lototsky ${ }^{1}$, Boris L. RozovskiI ${ }^{2}$ and Xiaoliang Wan $^{3}$
}

\begin{abstract}
This paper discusses analytical and numerical issues related to elliptic equations with random coefficients which are generally nonlinear functions of white noise. Singularity issues are avoided by using the Itô-Skorohod calculus to interpret the interactions between the coefficients and the solution. The solution is constructed by means of the Wiener Chaos (Cameron-Martin) expansions. The existence and uniqueness of the solutions are established under rather weak assumptions, the main of which requires only that the expectation of the highest order (differential) operator is a non-degenerate elliptic operator. The deterministic coefficients of the Wiener Chaos expansion of the solution solve a lower-triangular system of linear elliptic equations (the propagator). This structure of the propagator insures linear complexity of the related numerical algorithms. Using the lower triangular structure and linearity of the propagator, the rate of convergence is derived for a spectral $/ h p$ finite element approximation. The results of related numerical experiments are presented.
\end{abstract}

Mathematics Subject Classification. 35R60, 65L60, 60H15, 60H35.

Received July 3rd, 2009.

Published online August 26, 2010.

\section{INTRODUCTION}

The objective of this paper is to study, both analytically and numerically, linear elliptic equations with random coefficients. The perturbations introduced by the random coefficients can represent actual physical input or uncertainty about the model. In this paper, we limit our considerations to coefficients that are (possibly nonlinear) functions of Gaussian random fields. A popular example of an equation with random coefficients is Darcy equation for pressure with lognormal permeability field (see [8] and the references therein). It can be specified as the following Dirichlet problem:

$$
-\nabla(a(\boldsymbol{x}, \omega) \nabla u(\boldsymbol{x}))=f(\boldsymbol{x}), \boldsymbol{x} \in \mathcal{O} \subset \mathbb{R}^{d}, u_{\mid \partial \mathcal{O}}=g(\boldsymbol{x}),
$$

where

$$
a(\boldsymbol{x}, \omega)=\bar{a}(\boldsymbol{x}) \exp \left\{\epsilon(\boldsymbol{x})-\frac{1}{2} \mathbb{E}|\epsilon(\boldsymbol{x})|^{2}\right\},
$$

\footnotetext{
Keywords and phrases. Elliptic PDE, random coefficients, Wiener Chaos, spectral finite elements.

1 Department of Mathematics, USC, Los Angeles, CA 90089, USA. lototsky@math.usc.edu; http://www-rcf.usc.edu/ lototsky

2 Division of Applied Mathematics, Brown University, Providence, RI 02912, USA. rozovsky@dam.brown.edu

${ }^{3}$ Department of Mathematics, Center for Computation and Technology, Louisiana State University, Baton Rouge, LA 70803, USA. xlwan@math.lsu.edu 
$\omega$ symbolizes "chance", $\epsilon(\boldsymbol{x})=\sum_{k>1} \sigma_{k}(\boldsymbol{x}) \xi_{k}$ is the noise term, $\sigma_{k}(\boldsymbol{x})$ are deterministic functions, and $\boldsymbol{\xi}:=$ $\left\{\xi_{k}(\omega)\right\}_{k \geq 1}$ is a set of uncorrelated Gaussian random variables with zero mean and unit variance. The force $f$ and the boundary value $g$ as well as the boundary itself are assumed to be non-random. Clearly, $\epsilon(\boldsymbol{x})$ is a Gaussian random field with zero mean and covariance $Q(\boldsymbol{x}, \boldsymbol{y})=\sum_{k} \sigma_{k}(\boldsymbol{x}) \sigma_{k}(\boldsymbol{y})$ and $\bar{a}(\boldsymbol{x})$ is the mean of $a(\boldsymbol{x}, \omega): \bar{a}(\boldsymbol{x})=\mathbb{E}[a(\boldsymbol{x}, \omega)]$.

Another typical example is (1.1) with

$$
a(\boldsymbol{x}, \omega):=\bar{a}(\boldsymbol{x})+\epsilon(\boldsymbol{x}, \omega) .
$$

Models (1.1)-(1.3) have been actively investigated recently in the context of uncertainty quantification problems (see e.g. [2]). Note that equation (1.1) with the function $a(\boldsymbol{x}, \omega)$ from (1.3) is ill-posed because $a(\boldsymbol{x}, \omega)$ can take negative values.

Linear equation with multiplicative noise are usually referred to as bi-linear. These equations are much more challenging than linear equations with additive random perturbations. In fact, equation (1.1) is often too singular to be "physical". By this reason, certain regularization is in order for each of these two models.

One effective and popular regularization procedure for these models is to replace equation by its Wick product version

$$
-\nabla(a(\boldsymbol{x}, \omega) \diamond \nabla u(\boldsymbol{x}))=f(\boldsymbol{x}), \boldsymbol{x} \in \mathcal{O} \subset \mathbb{R}^{d}, u_{\mid \partial \mathcal{O}}=g(\boldsymbol{x}) ;
$$

see [8] for a discussion of the Wick product as a modeling tool. Admittedly, "Wick product" is anything but product, it is rather a stochastic convolution. In fact, Wick product is a version of Malliavin divergence operator. For example, $a(\boldsymbol{x}, \omega) \diamond \nabla u(\boldsymbol{x})$ is the action of the Malliavin divergence operator with respect to $a(\boldsymbol{x}, \omega)$ on the random field $\nabla u(\boldsymbol{x})$.

The idea of this regularization procedure was championed by Itô in his seminal work [9]. Specifically, Itô has replaced the "product" model

by a "stochastic convolution" model

$$
\dot{u}(t)=\mathbf{a}(u(t))+\mathbf{b}(u(t)) \cdot \dot{W}(t)
$$

$$
\dot{u}(t)=\mathbf{a}(u(t))+\mathbf{b}(u(t)) \diamond \dot{W}(t),
$$

which turned out to be equivalent to the stochastic Itô differential:

$$
\mathbf{b}(u(t)) \diamond \dot{W} \mathrm{~d} t=\mathbf{b}(u(t)) \mathrm{d} W(t) .
$$

In this paper we extend Itô's approach to elliptic stochastic PDEs. Since absence of the time variable prevents elliptic SPDEs from being "causal", the underlying Itô integrals are replaced by the Skorohod integrals (see [15]). The subsequent analysis of the equation is based on the Wiener chaos expansion (WCE) with respect to the Cameron-Martin basis. The Cameron-Martin basis consists of random variables

$$
\xi_{\alpha}=\prod_{k} \frac{\mathrm{H}_{\alpha_{k}}(\boldsymbol{\xi})}{\sqrt{\alpha_{k} !}}
$$

where $\boldsymbol{\alpha}=\left(\alpha_{1}, \alpha_{2}, \ldots\right)$ is a multi-index with non-negative integer entries and $\mathrm{H}_{\alpha_{k}}(x)$ is the Hermite polynomial of order $\alpha_{k}$ (see Sect. 2 for more detail). The WCE solution of the equation is given by the series $u(\boldsymbol{x})=$ $\sum_{\boldsymbol{\alpha} \in \mathcal{J}} u_{\boldsymbol{\alpha}}(\boldsymbol{x}) \xi_{\boldsymbol{\alpha}}$, where $u_{\boldsymbol{\alpha}}=\mathbb{E}\left[u \xi_{\boldsymbol{\alpha}}\right]$. One can view the Cameron-Martin expansion as a Fourier expansion that separates random and deterministic components of the equation. We demonstrate that the deterministic components $u_{\boldsymbol{\alpha}}(\boldsymbol{x})$ are uniquely defined by a lower triangular system of linear deterministic elliptic equations (see (3.5) below). We refer to this system as the (uncertainty) propagator. Since the basis elements $\xi_{\boldsymbol{\alpha}}$ are given by explicit formulas, the propagator is the key element of the solution.

From the numerical stand point, it is important that the lower triangular (in fact, bi-diagonal) structure of the propagator ensures linear computational complexity. While it is possible to define an approximate WCE solution for the standard multiplicative model (1.1), the corresponding propagator turns out a full system. 
From the statistical point of view, it is important that a solution of the Wick product model (1.4) is an unbiased random perturbation of a solution of the deterministic equation (1.5)

$$
-\nabla(\mathbb{E}[a(\boldsymbol{x}, \omega)] \nabla v(\boldsymbol{x}))=f(\boldsymbol{x}), \boldsymbol{x} \in \mathcal{O} \subset \mathbb{R}^{d}, u_{\mid \partial \mathcal{O}}=g(\boldsymbol{x})
$$

in that the mean (expectation) of the solution of equation (1.4) is a solution of equation (1.5).

In this paper we investigate elliptic equations of arbitrary deterministic and stochastic order. As usual, by deterministic order we understand the order of the highest differential operator; in particular, equation (1.1) has deterministic order two. The "stochastic order" of the equation is, by definition, the highest order of the Hermite polynomial in the Wiener chaos expansion of the random coefficients. For equation (1.1) with the coefficient $a(\boldsymbol{x}, \omega)$ defined by (1.3) the stochastic order is 1 ; for the same equation with the log-normal coefficient (1.2) this order is infinite. Note that any $a(\boldsymbol{x}, \omega)$ which is nonlinear function of $\boldsymbol{\xi}:=\left\{\xi_{k}(\omega)\right\}_{k \geq 1}$ has stochastic order higher than 1 .

The stochastic order is an important and also intricate characteristic of the equations in question. For example, analytically, problem (1.1) with Gaussian $a(\boldsymbol{x}, \omega)$ (stochastic order 1) is much more difficult than the lognormal $a(\boldsymbol{x}, \omega)$ (stochastic order $\infty$ ). One reason for this is that in the Gaussian case problem (1.1) is ill posed (from the classical point of view). However, as we will see later, this ill posedness is irrelevant in our setting. In particular, it is shown in Section 3 that bilinear stochastic elliptic PDEs are uniquely solvable under a few simple conditions. The key is the following:

Assumption A. The expectation of the highest order (differential) operator is a nondegenerate elliptic operator.

For example, in the setting (1.1) this means $\mathbb{E}[a(\boldsymbol{x}, \omega)] \geq c>0$.

In Section 4 it is shown that under assumptions that are very close to those that guarantee the existence and uniqueness of the solution one can construct effective finite-element approximation algorithms. More specifically, using the lower triangular structure and the linearity of the uncertainty propagator and incorporating the estimates of the operator norms, we provide an a priori error estimate for the convergence of spectral/hp finite element method. For equations of stochastic order one, this analysis has been carried out in [22]. The same strategy can be replicated to obtain new a priori error estimates for other numerical methods for solving the propagator.

As mentioned above the WCE solution is given by the series

$$
u(\boldsymbol{x})=\sum_{\boldsymbol{\alpha} \in \mathcal{J}} u_{\boldsymbol{\alpha}}(\boldsymbol{x}) \mathrm{H}_{\boldsymbol{\alpha}}(\boldsymbol{\xi}) / \sqrt{\boldsymbol{\alpha} !}
$$

However, very simple examples (see Sect. 3) demonstrate that the variance of WCE solutions is typically infinite or, more precisely, that $\mathbb{E}\|u\|^{2}=\sum_{\boldsymbol{\alpha} \in \mathcal{J}}\left\|u_{\boldsymbol{\alpha}}\right\|^{2}=\infty$. Fortunately, the blow-up of the Wick product model is controllable, in that the WCE solution can be effectively rescaled by simple weights $r_{\boldsymbol{\alpha}}$ such that $\sum_{\boldsymbol{\alpha}} r_{\boldsymbol{\alpha}}^{2} u_{\boldsymbol{\alpha}}^{2}<\infty$ (see Thm. 3.3). We remark that, in the "standard product" setting, the blow-up of the WCE solution is typically much more severe than in the Wick product setting (see Sect. 5).

There are alternative ways to address bi-linear elliptic SPDEs. The most developed alternative approach is based on Hida's white noise analysis [7]. The white noise approach exploits the built-in set of stochastic spaces, such as Hida or Kondratiev spaces [11,12], or even larger exponential spaces [16]. The traditional approach $[17,20,21]$, etc., has to select a stochastic space and then to study the largest possible class of equations admitting a solution in that space. The difference of our approach is that we select the stochastic space that is in some sense optimal for the particular equation under consideration.

The paper consists of four sections. Section 2 describes the classes of solution spaces for the equations of interest. The theorem about existence and uniqueness of the solution is in Section 3. Numerical analysis of the equation using finite-element approximation, including the proof of convergence, is in Section 4. Finally, Section 5 presents results of numerical experiments. 


\section{Weighted ChaOs SPACES}

Let $\mathcal{J}$ be the set of multi-indices $\boldsymbol{\alpha}=\left(\alpha_{1}, \alpha_{2}, \ldots\right)$ such that $\alpha_{k} \in\{0,1,2, \ldots\}$. For $\boldsymbol{\alpha}, \boldsymbol{\beta} \in \mathcal{J}$, we define

$$
|\boldsymbol{a}|=\sum_{k \geq 1} \alpha_{k}, \quad \boldsymbol{\alpha} !=\prod_{k \geq 1} \alpha_{k} !, \quad \boldsymbol{\alpha}+\boldsymbol{\beta}=\left(\alpha_{1}+\beta_{1}, \alpha_{2}+\beta_{2}, \ldots\right) .
$$

By definition, $\boldsymbol{\alpha}>0$ if $|\boldsymbol{\alpha}|>0$ and $\boldsymbol{\beta} \leq \boldsymbol{\alpha}$ if

$$
\beta_{k} \leq \alpha_{k} \text { for all } k \geq 1
$$

If $\boldsymbol{\beta} \leq \boldsymbol{\alpha}$, then we define

$$
\boldsymbol{\alpha}-\boldsymbol{\beta}=\left(\alpha_{1}-\beta_{1}, \alpha_{2}-\beta_{2}, \ldots\right)
$$

We use the following notations for the special multi-indices:

(1) (0) is the multi-index with all zero entries: $(\mathbf{0})_{k}=0$ for all $k$;

(2) $\boldsymbol{\epsilon}(\boldsymbol{i})$ is the multi-index of length 1 and with the single non-zero entry at position $i$ :

$$
\boldsymbol{\epsilon}(\boldsymbol{i})_{k}= \begin{cases}1, & \text { if } k=i \\ 0, & \text { if } k \neq i\end{cases}
$$

We also use convention $\boldsymbol{\epsilon}(\mathbf{0})=(\mathbf{0})$.

Given a sequence $q=\left(q_{k}, k \geq 1\right)$ of positive numbers and $\boldsymbol{\alpha} \in \mathcal{J}$, we define

$$
q^{\alpha}=\prod_{k \geq 1} q_{k}^{\alpha_{k}}
$$

Next, we introduce the following objects:

(1) $\mathbb{F}=(\Omega, \mathcal{F}, \mathbb{P})$, a complete probability space that is big enough to support countably many independent Gaussian random variables;

(2) $\boldsymbol{\xi}=\left\{\xi_{k}, k \geq 1\right\}$, i.i.d. standard normal random variables on $\mathbb{F}$;

(3) $V$, a real separable Hilbert space;

(4) $\mathrm{H}_{n}=\mathrm{H}_{n}(t), n=0,1,2, \ldots, t \in \mathbb{R}$, one-dimensional Hermite polynomial of order $n$ :

$$
\mathrm{H}_{n}(t)=(-1)^{n} \mathrm{e}^{t^{2} / 2} \frac{\mathrm{d}^{n}}{\mathrm{~d} t^{n}} \mathrm{e}^{-t^{2} / 2}
$$

In particular, $\mathrm{H}_{0}(t)=1, \mathrm{H}_{1}(t)=t, \mathrm{H}_{2}(t)=t^{2}-1, \mathrm{H}_{3}(t)=t^{3}-3 t, \ldots$

(5) Stochastic Hermite polynomials

$$
\mathrm{H}_{\boldsymbol{\alpha}}(\boldsymbol{\xi})=\prod_{k \geq 1} \mathrm{H}_{\alpha_{k}}\left(\xi_{k}\right), \quad \boldsymbol{\alpha} \in \mathcal{J}
$$

For example, if $\boldsymbol{\alpha}=(0,2,0,1,3,0,0, \ldots)$, then

$$
\mathrm{H}_{\boldsymbol{\alpha}}(\boldsymbol{\xi})=\left(\xi_{2}^{2}-1\right) \xi_{4}\left(\xi_{5}^{3}-3 \xi_{5}\right) .
$$

Recall the following result (Cameron and Martin [3]).

Theorem 2.1. The collection

$$
\left\{\frac{\mathrm{H}_{\boldsymbol{\alpha}}(\boldsymbol{\xi})}{\sqrt{\boldsymbol{\alpha} !}}, \alpha \in \mathcal{J}\right\}
$$


is an orthonormal basis in the space of square-integrable random variables that are measurable with respect to the $\sigma$-algebra generated by the collection $\boldsymbol{\xi}$.

In what follows, assume that the $\sigma$-algebra $\mathcal{F}$ in $\mathbb{F}$ is generated by $\boldsymbol{\xi}$, and denote by $L_{2}(\mathbb{F} ; V)$ the collection of square-integrable $V$-valued random elements. By Theorem 2.1, every $v \in L_{2}(\mathbb{F} ; V)$ has a unique representation

$$
v=\sum_{\boldsymbol{\alpha} \in \mathcal{J}} v_{\boldsymbol{\alpha}} \mathrm{H}_{\boldsymbol{\alpha}}(\boldsymbol{\xi}),
$$

where

$$
v_{\boldsymbol{\alpha}}=\frac{\mathbb{E}\left[v \mathrm{H}_{\boldsymbol{\alpha}}(\boldsymbol{\xi})\right]}{\boldsymbol{\alpha} !},
$$

and

$$
\mathbb{E}\|v\|_{V}^{2}=\sum_{\boldsymbol{\alpha} \in \mathcal{J}} \boldsymbol{\alpha} !\left\|v_{\boldsymbol{\alpha}}\right\|_{V}^{2} .
$$

Then, given a collection $\mathcal{R}=\left\{r_{\boldsymbol{\alpha}}, \boldsymbol{\alpha} \in \mathcal{J}\right\}$ of positive real numbers, we define the space $\mathcal{R} L_{2}(\mathbb{F} ; V)$ as the closure of $L_{2}(\mathbb{F} ; V)$ in the norm

$$
\|v\|_{\mathcal{R} L_{2}(\mathbb{F} ; V)}^{2}=\sum_{\boldsymbol{\alpha} \in \mathcal{J}} r_{\boldsymbol{\alpha}} \boldsymbol{\alpha} !\left\|v_{\boldsymbol{\alpha}}\right\|_{V}^{2} .
$$

The space $\mathcal{R} L_{2}(\mathbb{F} ; V)$ is called a weighted chaos space. We use the notation

$$
\mathcal{R} L_{2}(\mathbb{F})=\mathcal{R} L_{2}(\mathbb{F} ; \mathbb{R}) .
$$

Proposition 2.2. Let $\left\{v_{\boldsymbol{\alpha}}, \boldsymbol{\alpha} \in \mathcal{J}\right\}$ be a collection of elements from $V$. Then there exists a collection $\mathcal{R}$ such that $\sum_{\boldsymbol{\alpha} \in \mathcal{J}} v_{\boldsymbol{\alpha}} \mathrm{H}_{\boldsymbol{\alpha}}(\boldsymbol{\xi}) \in \mathcal{R} L_{2}(\mathbb{F} ; V)$.

Proof. We use the following result: if $p>1$, then

$$
\sum_{\alpha \in \mathcal{J}} \frac{1}{(2 \mathbb{N})^{p \alpha}}<\infty
$$

where $\mathbb{N}$ is the sequence of positive integers and

$$
(2 \mathbb{N})^{p \boldsymbol{\alpha}}=\prod_{k \geq 1}(2 k)^{p \alpha_{k}}
$$

(see [8], Prop. 7.1). Then it is enough to take

$$
r_{\boldsymbol{\alpha}}=\frac{1}{\alpha !(2 \mathbb{N})^{2 \boldsymbol{\alpha}}\left(1+\left\|v_{\boldsymbol{\alpha}}\right\|_{V}^{2}\right)} .
$$

Definition 2.3. A generalized random element is a collection $\left\{v_{\boldsymbol{\alpha}}, \boldsymbol{\alpha} \in \mathcal{J}\right\}$ of elements from $V$.

\section{Stochastic elliptic equations: Existence and uniqueness of solutions}

Let $\mathcal{I}$ be a subset of $\mathcal{J}$ such that $(0) \in \mathcal{I}$ and let $V^{\prime}$ be a real separable Hilbert space. Consider a collection $\left\{\mathrm{A}_{\boldsymbol{\alpha}}, \boldsymbol{\alpha} \in \mathcal{I}\right\}$ of bounded linear operators from $V$ to $V^{\prime}$, such that the operator $\mathrm{A}_{(\mathbf{0})}$ has a bounded inverse. The objective of this section is to study the existence and uniqueness of solution of the following stochastic equation

$$
\sum_{\boldsymbol{\beta} \in \mathcal{I}}\left(\mathrm{A}_{\boldsymbol{\beta}} u\right) \diamond \mathrm{H}_{\boldsymbol{\beta}}(\boldsymbol{\xi})=f
$$


where $f \in V^{\prime}$ is non-random and $\diamond$ denotes the Wick product [8]. In particular,

$$
\mathrm{H}_{\boldsymbol{\alpha}}(\boldsymbol{\xi}) \diamond \mathrm{H}_{\boldsymbol{\beta}}(\boldsymbol{\xi})=\mathrm{H}_{\boldsymbol{\alpha}+\boldsymbol{\beta}}(\boldsymbol{\xi}) .
$$

Definition 3.1. Equation (3.1) is said to have finite stochastic order $N$ if

$$
\max _{\boldsymbol{\alpha} \in \mathcal{I}}|\boldsymbol{\alpha}|=N
$$

The equation (3.1) is said to have infinite stochastic order if

$$
\sup _{\boldsymbol{\alpha} \in \mathcal{I}}|\boldsymbol{\alpha}|=\infty
$$

A special case of $(3.1)$ is

$$
\mathrm{A} u+\sum_{k \geq 1} \mathrm{M}_{k} u \diamond \xi_{k}=f
$$

this equations is studied in [14]. In (3.3), $\mathrm{A}_{\boldsymbol{\alpha}}=0$ for $|\boldsymbol{\alpha}|>1$, and the equation has stochastic order 1:

$$
\mathrm{A}_{(0)} u+\sum_{k \geq 1}\left(\mathrm{~A}_{\epsilon(\boldsymbol{k})} u\right) \diamond \mathrm{H}_{\boldsymbol{\epsilon}(\boldsymbol{k})}(\boldsymbol{\xi})=f .
$$

In general, (3.1) can be written as

$$
\mathrm{A}_{(0)} u=f-\sum_{\boldsymbol{\beta} \in \mathcal{I},|\boldsymbol{\beta}|>0}\left(\mathrm{~A}_{\boldsymbol{\beta}} u\right) \diamond \mathrm{H}_{\boldsymbol{\beta}}(\boldsymbol{\xi})
$$

and interpreted as a bi-linear stochastic perturbation of the deterministic equation $\mathrm{A}_{(0)} u=f$; this is the reason for assuming that $(\mathbf{0}) \in \mathcal{I}$. The use of the Wick product ensures that the perturbation has zero average.

In view of Proposition 2.2 and property (3.2) of the Hermite polynomials, the following definition is natural.

Definition 3.2. A solution of equation (3.1) is a generalized random element such that

$$
\begin{aligned}
\mathrm{A}_{(\mathbf{0})} u_{(0)} & =f,|\boldsymbol{\alpha}|=0, \\
\mathrm{~A}_{(\mathbf{0})} u_{\boldsymbol{\alpha}} & =-\sum_{\substack{\boldsymbol{\beta} \in \mathcal{I} \\
0<\boldsymbol{\beta} \leq \boldsymbol{\alpha}}} \mathrm{A}_{\boldsymbol{\beta}} u_{\boldsymbol{\alpha}-\boldsymbol{\beta}},|\boldsymbol{\alpha}|>0 .
\end{aligned}
$$

Indeed, writing $u=\sum_{\boldsymbol{\alpha} \in \mathcal{J}} u_{\boldsymbol{\alpha}} \mathrm{H}_{\boldsymbol{\alpha}}(\xi)$, substituting it formally into (3.1), and using (3.2), we find

$$
\sum_{\boldsymbol{\alpha} \in \mathcal{J}} \sum_{\boldsymbol{\beta} \in \mathcal{I}} \mathrm{A}_{\boldsymbol{\beta}} u_{\boldsymbol{\alpha}} \mathrm{H}_{\boldsymbol{\alpha}+\boldsymbol{\beta}}(\boldsymbol{\xi})=f
$$

or

$$
\sum_{\boldsymbol{\alpha} \in \mathcal{J}}\left(\sum_{\substack{\boldsymbol{\beta} \in \mathcal{I} \\ \boldsymbol{\beta} \leq \boldsymbol{\alpha}}} \mathrm{A}_{\boldsymbol{\beta}} u_{\boldsymbol{\alpha}-\boldsymbol{\beta}}\right) \mathrm{H}_{\boldsymbol{\alpha}}(\boldsymbol{\xi})=f .
$$

Equating the coefficients of every $\mathrm{H}_{\boldsymbol{\alpha}}(\boldsymbol{\xi})$, we conclude that (3.5) must hold.

The following theorem establishes the existence and uniqueness of solution of equation (3.1). 
Theorem 3.3. Assume that:

(1) The operator $\mathrm{A}_{(\mathbf{0})}$ has a bounded inverse $\mathrm{A}_{(\mathbf{0})}^{-1}$ from $V^{\prime}$ to $V$.

(2) Each of the operators $\mathrm{A}_{(\mathbf{0})}^{-1} \mathrm{~A}_{\boldsymbol{\beta}}, \boldsymbol{\beta} \in \mathcal{I},|\boldsymbol{\beta}|>0$, is bounded on $V$, with the operator norm $C_{\boldsymbol{\beta}}$.

(3) There exists a sequence $b=\left(b_{1}, b_{2}, \ldots\right)$ of positive numbers, such that, for all $\boldsymbol{\beta} \in \mathcal{I}$,

$$
C_{\boldsymbol{\beta}} \leq b^{\boldsymbol{\beta}}
$$

(4) The function $f$ is deterministic and belongs to $V^{\prime}$.

Then

(1) Equation (3.1) has a unique solution $u$;

(2) The solution is an element of the weighted chaos space $\mathcal{R} L_{2}(\mathbb{F} ; V)$ with

$$
r_{\boldsymbol{\alpha}}=\frac{c^{\alpha}}{\alpha !}
$$

for some sequence $c=\left(c_{1}, c_{2}, \ldots\right)$ of positive numbers.

Proof. To begin, let us first understand the structure of the system of equations (3.5). Define

$$
\mathrm{B}_{\boldsymbol{\beta}}=-\mathrm{A}_{(0)}^{-1} \mathrm{~A}_{\boldsymbol{\beta}}, \boldsymbol{\beta} \in \mathcal{I}
$$

Then (3.5) becomes

$$
\begin{aligned}
u_{(0)} & =\mathrm{A}_{(\mathbf{0})}^{-1} f,|\boldsymbol{\alpha}|=0, \\
u_{\boldsymbol{\alpha}} & =\sum_{\substack{\boldsymbol{\beta} \in \mathcal{I} \\
0<\boldsymbol{\beta} \leq \boldsymbol{\alpha}}} \mathrm{B}_{\boldsymbol{\beta}} u_{\boldsymbol{\alpha}-\boldsymbol{\beta}},|\boldsymbol{\alpha}|>0 .
\end{aligned}
$$

Thus, $u_{\boldsymbol{\alpha}}$ with $|\boldsymbol{\alpha}|=n>0$ is determined by $u_{\boldsymbol{\alpha}}$ with $|\boldsymbol{\alpha}|<n$, which implies existence and uniqueness of solution of (3.5), and hence of (3.1).

In view of (2.7), to prove that the solution is an element of a weighted chaos space with weights $r_{\boldsymbol{\alpha}}$ of the form $r_{\boldsymbol{\alpha}}=c^{\boldsymbol{\alpha}} / \boldsymbol{\alpha}$ ! for some sequence $c=\left(c_{1}, c_{2}, \ldots\right)$ of positive numbers, it is enough to show that there exists a sequence of positive numbers $q=\left(q_{k}, k \geq 1\right)$ such that

$$
\left\|u_{\boldsymbol{\alpha}}\right\|_{V} \leq q^{\boldsymbol{\alpha}}\left\|u_{(0)}\right\|_{V}
$$

then we can take $c_{k}=\left(2 k q_{k}\right)^{-2}$. Thus, to complete the proof, it remains to establish (3.8).

We start with the particular case (3.3). Even though this equation has been studied in [14], the analysis there relies on a closed-form expression for $u_{\boldsymbol{\alpha}}$ and does not easily extend to higher orders. Denote by $\mathrm{B}_{k}$ the operator $\mathrm{A}^{-1} \mathrm{M}_{k}$. Then, for $|\boldsymbol{\alpha}|>0$, (3.7) becomes

$$
u_{\boldsymbol{\alpha}}=\sum_{k \geq 1} \mathbf{1}_{\alpha_{k}>0} \mathrm{~B}_{k} u_{\boldsymbol{\alpha}-\epsilon(k)}
$$

We now show by induction that (3.8) holds with

$$
q_{k}=4 k^{2} b_{k}
$$


Since $u_{\boldsymbol{\epsilon}(\boldsymbol{k})}=\mathrm{B}_{k} u_{(\mathbf{0})}$, we have (3.8) for $|\boldsymbol{\alpha}|=1$. Now assume that (3.8) holds for all $\boldsymbol{\alpha}$ with $|\boldsymbol{\alpha}| \leq n-1$. Then (3.9) and the triangle inequality imply, for $\boldsymbol{\alpha}$ with $|\boldsymbol{\alpha}|=n$,

$$
\begin{aligned}
\left\|u_{\boldsymbol{\alpha}}\right\|_{V} & \leq\left\|u_{(\mathbf{0})}\right\|_{V} \sum_{k \geq 1} \mathbf{1}_{\alpha_{k}>0} b_{k} q^{\boldsymbol{\alpha}-\boldsymbol{\epsilon}(\boldsymbol{k})} \\
& =q^{\boldsymbol{\alpha}}\left\|u_{(\mathbf{0})}\right\|_{V} \sum_{k \geq 1} \frac{\mathbf{1}_{\alpha_{k}>0}}{4 k^{2}} \leq q^{\boldsymbol{\alpha}}\left\|u_{(\mathbf{0})}\right\|_{V} \sum_{k=1}^{\infty} \frac{1}{4 k^{2}} \\
& <q^{\boldsymbol{\alpha}}\left\|u_{(\mathbf{0})}\right\|_{V},
\end{aligned}
$$

because $\sum_{k \geq 1} k^{-2}=\left(\pi^{2} / 6\right)<4$. This completes the proof of (3.8) for equation (3.3).

Let us now consider the general equation (3.1). We will use assumption (3.6) to show that (3.8) holds for $N>1$ with

where

$$
q_{k}=C_{0} k^{2} b_{k}
$$

$$
C_{0}=2 \sum_{\alpha \in \mathcal{J}} \frac{1}{(2 \mathbb{N})^{2 \alpha}}
$$

see (2.7). For the curious reader, we note that numerical computations show that $C_{0} \approx 3.2$, and simple analytic considerations put the value of $C_{0}$ in the range $[3.0,3.4]$.

As in the case of equation (3.3), the proof of (3.8) is by induction on $|\alpha|$. Let $k_{0}=\min \{|\beta|: \beta \in \mathcal{I}\}$. If $|\boldsymbol{\alpha}| \leq k_{0}$, then $u_{\boldsymbol{\alpha}}=0$ and so (3.8) trivially holds. If $\boldsymbol{\alpha} \in \mathcal{I}$ and $|\boldsymbol{\alpha}|=k_{0}$, then (3.5) implies

$$
u_{\boldsymbol{\alpha}}=\mathrm{B}_{\boldsymbol{\alpha}} u_{(\mathbf{0})}, \quad\left\|u_{\boldsymbol{\alpha}}\right\| \leq b^{\boldsymbol{\alpha}}\left\|u_{(\mathbf{0})}\right\|_{V} \leq q^{\boldsymbol{\alpha}}\left\|u_{(\mathbf{0})}\right\|_{V} .
$$

Now assume that (3.8) holds for all $u_{\boldsymbol{\alpha}}$ with $|\alpha| \leq n-1$ and fix $|\boldsymbol{\alpha}|=n>k_{0}$. By (3.7), the triangle inequality, and the induction assumption,

$$
\begin{aligned}
\left\|u_{\boldsymbol{\alpha}}\right\|_{V} & \leq \sum_{\substack{\boldsymbol{\beta} \in \mathcal{I} \\
0<\boldsymbol{\beta} \leq \boldsymbol{\alpha}}}\left\|\mathrm{B}_{\boldsymbol{\beta}} u_{\boldsymbol{\alpha}-\boldsymbol{\beta}}\right\|_{V} \leq\left\|u_{(\mathbf{0})}\right\|_{V} \frac{q^{\boldsymbol{\alpha}}}{C_{0}} \sum_{\boldsymbol{\beta} \in \mathcal{I}} \frac{1}{(2 \mathbb{N})^{2 \boldsymbol{\beta}}} \\
& \leq\left\|u_{(\mathbf{0})}\right\|_{V} \frac{q^{\boldsymbol{\alpha}}}{C_{0}} \sum_{\boldsymbol{\beta} \in \mathcal{J}} \frac{1}{(2 \mathbb{N})^{2 \boldsymbol{\beta}}} \leq q^{\boldsymbol{\alpha}}\left\|u_{(\mathbf{0})}\right\|_{V},
\end{aligned}
$$

where the last inequality follows from (3.10). This completes the proof of Theorem 3.3.

Below, we illustrate Theorem 3.3 on several examples. To emphasize the main points, we consider a simplified version of equation (3.1), in which:

(1) $V=V^{\prime}=\mathbb{R}$, so that every operator $\mathrm{A}_{\boldsymbol{\beta}}$ is multiplication by a real number; then, with no loss of generality, we take $\mathrm{A}_{(\mathbf{0})}=1$.

(2) $f=1$.

(3) The operators $\mathrm{A}_{\boldsymbol{\beta}}$ are such that $\mathrm{A}_{\boldsymbol{\beta}} \neq 0$ only if $\boldsymbol{\beta}=(n, 0,0, \ldots)$ for some $n=0,1,2, \ldots$

In other words, (3.1) becomes

$$
u=1+\sum_{k \geq 1} a_{k} u \diamond \mathrm{H}_{k}(\xi),
$$

where $\xi$ is a standard Gaussian random variable. The solution $u$ has the form

$$
u=\sum_{n \geq 0} u_{n} \mathrm{H}_{n}(\xi)
$$


for some real numbers $u_{n}$, and

$$
\mathbb{E}|u|^{2}=\sum_{n \geq 1} n ! u_{n}^{2}
$$

As a first example, consider the equation

$$
u=1+u \diamond \xi
$$

It is easy to verify that $u=1+\sum_{k>1} \mathrm{H}_{k}(\xi)$ is a solution, that is, $u_{k}=1$ for all $k$. By $(3.12), u \notin L_{2}(\mathbb{F} ; \mathbb{R})$.

The coefficients of the solution of (3.13) are bounded. With a simple modification of (3.13), we can get coefficients that grow exponentially fast. Indeed, consider

$$
u=1+u \diamond \xi+u \diamond\left(\xi^{2}-1\right)
$$

recall that $\xi^{2}-1=\mathrm{H}_{2}(\xi)$. Then $(3.5)$ implies

$$
u_{0}=1, u_{1}=1, u_{n}=u_{n-1}+u_{n-2}, n \geq 2 .
$$

Thus, $u_{n}$ is $n$th Fibonacci number, and, as $n \rightarrow \infty, u_{n}$ behaves asymptotically as $(1+\sqrt{5})^{n} / 2^{n}$. In other words, we have $u_{n} \geq r^{n}$ for some $r>1$. Again, (3.12) implies $\mathbb{E} u^{2}=\infty$ and so $u \notin L_{2}(\mathbb{F} ; \mathbb{R})$.

Examples (3.13) and (3.14) show that, if equation (3.1) has finite stochastic order, then, in general, the solution does not belong to $L_{2}(\mathbb{F} ; V)$. In fact, we are not aware of any elliptic equation with multiplicative finite-order noise and with a square-integrable solution.

Let us consider now the equation

$$
u=1-\sum_{n \geq 1} \frac{u \diamond \mathrm{H}_{n}(\xi)}{n !} .
$$

This equation has infinite stochastic order. Direct computations show that

$$
u=1+\sum_{n \geq 1} \frac{(-1)^{n}}{n !} \mathrm{H}_{n}(\xi)=\mathrm{e}^{-\xi-(1 / 2)}
$$

one can also derive this by re-writing (3.15) as

$$
\mathrm{e}^{\xi-(1 / 2)} \diamond u=1
$$

and noticing that $\mathrm{e}^{\xi-(1 / 2)} \diamond \mathrm{e}^{-\xi-(1 / 2)}=1$. In particular, $\mathbb{E} u^{2}=e<\infty$, so $u \in L_{2}(\mathbb{F} ; \mathbb{R})$.

\section{Numerical analysis of a stochastic Dirichlet problem}

Let $\mathcal{O} \in \mathbb{R}^{d}$ be a bounded, open, connected set with a Lipschitz continuous boundary $\partial \mathcal{O}$. Denote by $\langle\cdot, \cdot\rangle$ the inner product in $L_{2}(\mathcal{O})$. Consider the following problem:

$$
\left\{\begin{aligned}
\mathrm{A} u+\sum_{\substack{\boldsymbol{\beta} \in \mathcal{I} \\
|\boldsymbol{\beta}| \geq 1}} \mathrm{~A}_{\boldsymbol{\beta}} u \diamond \mathrm{H}_{\boldsymbol{\beta}}(\boldsymbol{\xi})=f, & \boldsymbol{x} \in \mathcal{O}, \\
u=0, & \boldsymbol{x} \in \partial \mathcal{O},
\end{aligned}\right.
$$


where $\boldsymbol{\xi}$ is a finite- or infinite-dimensional standard Gaussian random variable. For simplicity, we assume that the force term $f(\boldsymbol{x})$ is a deterministic function. The operators $\mathrm{A}=\mathrm{A}_{(\mathbf{0})}$ and $\mathrm{A}_{\boldsymbol{\beta}}$ are defined by

$$
\begin{array}{r}
\mathrm{A} u(\boldsymbol{x})=-\sum_{i, j=1}^{d} D_{i}\left(a_{i j}(\boldsymbol{x}) D_{j} u(\boldsymbol{x})\right), \\
\mathrm{A}_{\boldsymbol{\beta}}(\boldsymbol{x})=w_{\boldsymbol{\beta}}(\boldsymbol{x}) \sum_{i, j=1}^{d} D_{i}\left(\sigma_{i j, \boldsymbol{\beta}}(\boldsymbol{x}) D_{j} u(\boldsymbol{x})\right),
\end{array}
$$

where the functions $a_{i j}(\boldsymbol{x}), \sigma_{i j, \boldsymbol{\beta}}(\boldsymbol{x})$, and $w_{\boldsymbol{\beta}}(\boldsymbol{x})$ satisfy the following assumptions:

\section{Assumption 4.1.}

(a) The functions $a_{i j}(\boldsymbol{x})$ are measurable and bounded in the closure $\overline{\mathcal{O}}$ of $\mathcal{O}$.

(b) There exist positive numbers $A_{1}, A_{2}$ such that $A_{1}|\boldsymbol{y}|^{2} \leq a_{i j}(\boldsymbol{x}) y_{i} y_{j} \leq A_{2}|\boldsymbol{y}|^{2}$ for all $\boldsymbol{x} \in \overline{\mathcal{O}}$ and $\boldsymbol{y} \in \mathbb{R}^{d}$.

(c) The functions $\sigma_{i j, \boldsymbol{\beta}}(\boldsymbol{x})$ are bounded and measurable:

$$
\left|\sigma_{i j, \boldsymbol{\beta}}(\boldsymbol{x})\right| \leq C_{\boldsymbol{\beta}}^{\sigma} \text { for all } \boldsymbol{x} \in \overline{\mathcal{O}}, i, j=1, \ldots, d .
$$

(d) The functions $w_{\boldsymbol{\beta}}(\boldsymbol{x})$ are bounded and Lipschitz continuous:

$$
\left|w_{\boldsymbol{\beta}}(\boldsymbol{x})\right| \leq C_{\boldsymbol{\beta}}^{w} \text { and }\left|w_{\boldsymbol{\beta}}(\boldsymbol{x})-w_{\boldsymbol{\beta}}(\boldsymbol{y})\right| \leq C_{\boldsymbol{\beta}}^{L}|\boldsymbol{x}-\boldsymbol{y}|, \quad \boldsymbol{x}, \boldsymbol{y} \in \overline{\mathcal{O}} .
$$

(e) There exists a sequence $b=\left(b_{1}, b_{2}, \ldots\right)$ of positive numbers such that, for all $\boldsymbol{\beta} \in \mathcal{I}$,

$$
\left(C_{\boldsymbol{\beta}}^{L}+C_{\boldsymbol{\beta}}^{w}\right) C_{\boldsymbol{\beta}}^{\sigma} \leq b^{\boldsymbol{\beta}} .
$$

Taking as $V$ and $V^{\prime}$ the Sobolev spaces $H_{0}^{1}(\mathcal{O})$ and $H^{-1}(\mathcal{O})$, respectively, we note that Theorem 3.3 applies to equation (4.1) because the operators $\mathrm{A}^{-1} \mathrm{~A}_{\boldsymbol{\beta}}$ are bounded on $V$. Indeed, note that

$$
\|u\|_{V}^{2}=\|u\|_{L_{2}(\mathcal{O})}^{2}+\|\nabla u\|_{L_{2}(\mathcal{O})}^{2}
$$

and let $C_{\pi}$ be the Poincaré constant for the domain $\mathcal{O}$ :

$$
\|u\|_{L_{2}(\mathcal{O})}^{2} \leq C_{\pi}\|\nabla u\|_{L_{2}(\mathcal{O})}^{2} .
$$

Assumption 4.1(b) implies

$$
\langle\mathrm{A} u, u\rangle \geq \frac{A_{1}}{1+C_{\pi}}\|u\|_{V}^{2}, u \in V
$$

here $\langle\cdot, \cdot\rangle$ denotes the duality paring between $V^{\prime}$ and $V$ relative to the inner product in $L_{2}(\mathcal{O})$. Then, by direct computation,

$$
\left\|\mathrm{A}^{-1} \mathrm{~A}_{\boldsymbol{\beta}} u\right\|_{V} \leq\left(\frac{1+C_{\pi}}{A_{1}}\right)\left(C_{\boldsymbol{\beta}}^{L}+C_{\boldsymbol{\beta}}^{w}\right) C_{\boldsymbol{\beta}}^{\sigma} .
$$

Example 4.2. Let $G(\boldsymbol{x})$ is a Gaussian field on $\mathcal{O}$ with representation

$$
G(\boldsymbol{x})=\sum_{k=1}^{\infty} h_{k}(\boldsymbol{x}) \xi_{k}
$$

where $\left\{h_{k}, k \geq 1\right\}$ are smooth bounded functions such that

$$
\sum_{k \geq 1}\left(\sup _{\boldsymbol{x} \in \mathcal{O}}\left|h_{k}(\boldsymbol{x})\right|^{2}+\sup _{\boldsymbol{x} \in \mathcal{O}}\left|\nabla h_{k}(\boldsymbol{x})\right|^{2}\right)<\infty
$$


and $\boldsymbol{\xi}=\left\{\xi_{k}, k \geq 1\right\}$ is a collection of independent standard Gaussian random variables. Note that

$$
\bar{G}(\boldsymbol{x}):=\mathbb{E} \mathrm{e}^{G(\boldsymbol{x})}=\exp \left(\frac{1}{2} \sum_{k \geq 1} h_{k}^{2}(\boldsymbol{x})\right) .
$$

Examples of (4.7) are Karhuan-Loève expansion [13], spectral expansion [19], and Fourier-Wavelet expansion [6].

Consider the equation

$$
\nabla \cdot\left(\mathrm{e}^{G(\boldsymbol{x})} \diamond \nabla u\right)=f .
$$

If

$$
\mathfrak{h}_{\boldsymbol{\alpha}}(\boldsymbol{x})=\frac{\bar{G}(\boldsymbol{x})}{\boldsymbol{\alpha} !} \prod_{k} h_{k}^{\alpha_{k}}(\boldsymbol{x}), \boldsymbol{\alpha} \in \mathcal{J}
$$

then

$$
\mathrm{e}^{G(\boldsymbol{x})}=\bar{G}(\boldsymbol{x})\left(1+\sum_{|\boldsymbol{\beta}| \geq 1} \mathfrak{h}_{\boldsymbol{\beta}}(\boldsymbol{x}) \mathrm{H}_{\boldsymbol{\beta}}(\boldsymbol{\xi})\right)
$$

and (4.9) becomes a particular case of (4.1):

$$
\nabla \cdot(\bar{G}(\boldsymbol{x}) \nabla u)+\sum_{|\boldsymbol{\beta}| \geq 1} \nabla \cdot\left(\bar{G}(\boldsymbol{x}) \mathfrak{h}_{\boldsymbol{\beta}}(\boldsymbol{x}) \nabla u\right) \diamond \mathrm{H}_{\boldsymbol{\beta}}(\boldsymbol{\xi})=f ;
$$

to satisfy Assumption 4.1(e), take $b_{k}=\sup _{\boldsymbol{x} \in \mathcal{O}}\left|h_{k}(\boldsymbol{x})\right|+\sup _{\boldsymbol{x} \in \mathcal{O}}\left|\nabla h_{k}(\boldsymbol{x})\right|$.

Replacing in (4.9) the usual exponential $\mathrm{e}^{G(\boldsymbol{x})}$ with the Wick exponential

$$
\mathrm{e}^{\diamond G((x))}=\mathrm{e}^{G(\boldsymbol{x})-\frac{1}{2} \sum_{k \geq 1} h_{k}^{2}(\boldsymbol{x})}
$$

also leads to an equation of the form (4.1).

The objective of this section is to study numerical solution of (4.1). Specifically, we construct an approximation of (4.1) using finite elements in the physical space $\mathcal{O}$ and a truncated chaos expansion in the probability space. For the sake of concreteness we assume that the physical space is two-dimensional: $d=2$.

Let $\mathscr{T}_{h}$ be a family of triangulations of $\mathcal{O}$ with straight edges and $h$ the maximum size of the elements in $\mathscr{T}_{h}$. We assume that the family is regular, in other words, the minimal angle of all the triangles is bounded from below by a positive constant. We define the finite element space as

$$
V_{h, p}^{K}=\left\{v \mid v \circ F_{K}^{-1} \in \mathscr{P}_{p}(R)\right\}, \quad V_{h, p}=\left\{v \in H^{1}(D)|v|_{K} \in V_{h, p}^{K}, K \in \mathscr{T}_{h}\right\},
$$

where $F_{K}$ is the mapping function for the element $K$ which maps the reference element $R$ (for example, an equilateral triangle or an isoceles right triangle) to the element $K$ and $\mathscr{P}_{p}(R)$ denotes the set of polynomials of degree at most $p$ on $R$. We assume that $\left.v_{h}\right|_{\partial \mathcal{O}}=0, v_{h} \in V_{h, p}$. Thus, $V_{h, p}$ is an approximation of $H_{0}^{1}(\mathcal{O})$ by piece-wise polynomial functions. There exist many choices of basis functions on the reference elements, such as $h$-type finite elements [5], spectral $/ h p$ elements [10,18], etc.

To describe truncated chaos expansion, fix positive integers $M, n$ and define the finite dimensional set $\mathcal{J}_{M, n} \subset \mathcal{J}$ as

$$
\mathcal{J}_{M, n}:=\left\{\boldsymbol{\alpha}|| \boldsymbol{\alpha} \mid \leq M, \alpha_{k}=0, k>n\right\} .
$$

The truncated Wiener chaos space $V^{M, n}$ is then

$$
V^{M, n}:=\left\{\sum_{\boldsymbol{\alpha} \in \mathcal{J}_{M, n}} f_{\boldsymbol{\alpha}} \mathrm{H}_{\boldsymbol{\alpha}}(\boldsymbol{\xi}) \mid f_{\boldsymbol{\alpha}} \in \mathbb{R}\right\} .
$$


Similarly, let

$$
\mathcal{I}_{M, n}=\mathcal{I} \cap \mathcal{J}_{M, n}
$$

It follows from (3.5) that $u_{\boldsymbol{\alpha}}$ is fully determined by coefficients $u_{\boldsymbol{\beta}}$ and operators $\mathrm{A}_{\boldsymbol{\beta}}$ with $\boldsymbol{\beta} \leq \boldsymbol{\alpha}$. Thus we only need to keep the operators $\mathrm{A}_{\boldsymbol{\beta}}$ with $\boldsymbol{\beta} \in \mathcal{I}_{M, n}$ if we only consider the coefficients $u_{\boldsymbol{\alpha}}$ with $\boldsymbol{\alpha} \in \mathcal{J}_{M, n}$.

The sFEM (stochastic finite element method) can be formulated as follows: Find $u_{h, p}^{M, n} \in V_{h, p} \otimes V^{M, n}$ such that, for all $v \in V_{h, p} \otimes V^{M, n}$,

$$
\mathbb{E}\left\langle\mathrm{A}_{(\mathbf{0})} u_{h, p}^{M, n}, v\right\rangle+\mathbb{E} \sum_{\substack{\boldsymbol{\beta} \in \mathcal{I}_{M, n} \\|\boldsymbol{\beta}|>0}}\left\langle\mathrm{~A}_{\boldsymbol{\beta}} u_{h, p}^{M, n} \diamond \mathrm{H}_{\boldsymbol{\beta}}(\boldsymbol{\xi}), v\right\rangle=\mathbb{E}\langle f, v\rangle .
$$

Remark 4.3. The underlying idea of sFEM is to consider a finite element approximation, followed by a truncation of the chaos expansion. Alternatively, it is possible to truncate the chaos expansion first and then apply the finite element method to the truncated version of the uncertainty propagator (3.5):

$$
\begin{aligned}
& \mathrm{A}_{(\mathbf{0})} u_{(\mathbf{0})}=f,|\boldsymbol{\alpha}|=0, \\
& \mathrm{~A}_{(\mathbf{0})} u_{\boldsymbol{\alpha}}+\sum_{\substack{\boldsymbol{\beta} \in \mathcal{I}_{M, n} \\
0<\boldsymbol{\beta} \leq \boldsymbol{\alpha}}} \mathrm{A}_{\boldsymbol{\beta}} u_{\boldsymbol{\alpha}-\boldsymbol{\beta}}=0,|\boldsymbol{\alpha}|>0 .
\end{aligned}
$$

This approach will not be discussed in this paper.

The following is the main result about the convergence of the stochastic finite element method.

Theorem 4.4. Assume that conditions given in Assumption 4.1 and Theorem 3.3 are satisfied and in addition, $u \in \mathcal{R} L_{2}\left(\mathbb{F} ; H^{m+1}(\mathcal{O})\right)$ for some integer $m \geq 1$. Then

$$
\left\|u-u_{h, p}^{M, n}\right\|_{\mathcal{R} L_{2}\left(\mathbb{F} ; H_{0}^{1}(\mathcal{O})\right)} \leq C_{1} h^{\mu-1} p^{-m}\|u\|_{\mathcal{R} L_{2}\left(\mathbb{F} ; H^{m+1}(\mathcal{O})\right)}+C_{2}\|f\|_{H^{-1}(\mathcal{O})}\left(\frac{1}{2^{M}}+\frac{1}{\sqrt{n}}\right),
$$

where $\mu=\min (m+1, p+1)$, the constants $C_{1}$ and $C_{2}$ are independent of $h$ and $p$, and $C_{2}$ is also independent of $M, n$.

Proof. Define $V=H_{0}^{1}(\mathcal{O})$ and let the Wiener-chaos expansion of $u$ and $u_{h, p}^{M, n}$ be

$$
u=\sum_{\boldsymbol{\alpha} \in \mathcal{J}} u_{\boldsymbol{\alpha}} \mathrm{H}_{\boldsymbol{\alpha}}(\boldsymbol{\xi}), u_{h, p}^{M, n}=\sum_{\boldsymbol{\alpha} \in \mathcal{J}_{M, n}} \hat{u}_{\boldsymbol{\alpha}} \mathrm{H}_{\boldsymbol{\alpha}}(\boldsymbol{\xi})
$$

respectively. Then

$$
u-u_{h, p}^{M, n}=\sum_{\boldsymbol{\alpha} \in \mathcal{J}_{M, n}}\left(u_{\boldsymbol{\alpha}}-\hat{u}_{\boldsymbol{\alpha}}\right) \mathrm{H}_{\boldsymbol{\alpha}}+\sum_{\boldsymbol{\alpha} \in \mathcal{J} \backslash \mathcal{J}_{M, n}} u_{\alpha} \mathrm{H}_{\boldsymbol{\alpha}},
$$

and

$$
\begin{aligned}
\left\|u-u_{h, p}^{M, n}\right\|_{\mathcal{R} L_{2}(\mathbb{F} ; V)}^{2}= & \sum_{\boldsymbol{\alpha} \in \mathcal{J}_{M, n}}\left\|u_{\boldsymbol{\alpha}}-\hat{u}_{\boldsymbol{\alpha}}\right\|_{V}^{2}\left\|\mathrm{H}_{\boldsymbol{\alpha}}\right\|_{\mathcal{R} L_{2}(\mathbb{F})}^{2} \\
& +\sum_{\boldsymbol{\alpha} \in \mathcal{J} \backslash \mathcal{J}_{M, n}}\left\|u_{\boldsymbol{\alpha}}\right\|_{V}^{2}\left\|\mathrm{H}_{\boldsymbol{\alpha}}\right\|_{\mathcal{R} L_{2}(\mathbb{F})}^{2}=I_{1}+I_{2} .
\end{aligned}
$$

The structure of equation (3.5) ensures that the coefficients $u_{\boldsymbol{\alpha}}$ with $\boldsymbol{\alpha} \in \mathcal{J} \backslash \mathcal{J}_{M, n}$ and operators $\mathrm{A}_{\boldsymbol{\beta}}$ with $\boldsymbol{\beta} \in \mathcal{I} \backslash \mathcal{I}_{M, n}$ do not affect $\hat{u}_{\boldsymbol{\alpha}}$ with $\boldsymbol{\alpha} \in \mathcal{J}_{M, n}$. In other words, $I_{1}$ is the error from the finite element discretization and $I_{2}$ is from the truncation of the chaos expansion. 
We first look at the term $I_{1}$ by considering equation (4.14). Since the equation for $u_{(0)}$ is a usual deterministic elliptic equation, we here only consider the equations for $u_{\boldsymbol{\alpha}}$ with $|\boldsymbol{\alpha}|>0$. By (4.13),

$$
\mathrm{A}_{(0)} \hat{u}_{\boldsymbol{\alpha}}+\sum_{\substack{\boldsymbol{\beta} \in \mathcal{I}_{M, n} \\ 0<\boldsymbol{\beta} \leq \boldsymbol{\alpha}}} \mathrm{A}_{\boldsymbol{\beta}} \hat{u}_{\boldsymbol{\alpha}-\boldsymbol{\beta}}=0 .
$$

Furthermore, although we truncate the number of random variables and the multi-index set $\mathcal{I}$, the structure of the uncertainty propagator (3.5) ensures that the exact solution also satisfied

$$
\mathrm{A}_{(0)} u_{\boldsymbol{\alpha}}+\sum_{\substack{\boldsymbol{\beta} \in \mathcal{I}_{M, n} \\ 0<\boldsymbol{\beta} \leq \boldsymbol{\alpha}}} \mathrm{A}_{\boldsymbol{\beta}} u_{\boldsymbol{\alpha}-\boldsymbol{\beta}}=0 .
$$

Denote by $a(\cdot, \cdot)$ the bilinear form generated by the elliptic operator $\mathrm{A}_{(\mathbf{0})}$ :

$$
a(u, v)=\langle\mathrm{A} u, v\rangle .
$$

Then, for every $v_{h} \in V_{h, p}$,

$$
\begin{aligned}
a\left(\hat{u}_{\boldsymbol{\alpha}}-v_{h}, \hat{u}_{\boldsymbol{\alpha}}-v_{h}\right) & =a\left(u_{\boldsymbol{\alpha}}-v_{h}, \hat{u}_{\boldsymbol{\alpha}}-v_{h}\right)+a\left(\hat{u}_{\boldsymbol{\alpha}}-u_{\boldsymbol{\alpha}}, \hat{u}_{\boldsymbol{\alpha}}-v_{h}\right) \\
& =a\left(u_{\boldsymbol{\alpha}}-v_{h}, \hat{u}_{\boldsymbol{\alpha}}-v_{h}\right)-\sum_{\substack{\boldsymbol{\beta} \in \mathcal{I}_{M, n} \\
0<\boldsymbol{\beta} \leq \boldsymbol{\alpha}}}\left\langle\mathrm{A}_{\boldsymbol{\beta}}\left(u_{\boldsymbol{\alpha}-\boldsymbol{\beta}}-\hat{u}_{\boldsymbol{\alpha}-\boldsymbol{\beta}}\right), \hat{u}_{\boldsymbol{\alpha}}-v_{h}\right\rangle .
\end{aligned}
$$

Assumption 4.1(b) implies

$$
\frac{A_{1}}{1+C_{\pi}}\|u\|_{V}^{2} \leq a(u, u), a(u, v) \leq A_{2}\|u\|_{V}\|v\|_{V}, u \in V
$$

where $C_{\pi}$ is the Poincaré constant for the domain $\mathcal{O}$; see (4.6). Then, with $C_{1}=A_{1} /\left(1+C_{\pi}\right)$ and thinking $w_{h}=\hat{u}_{\boldsymbol{\alpha}}-v_{h}$,

$$
C_{1}\left\|\hat{u}_{\boldsymbol{\alpha}}-v_{h}\right\|_{V} \leq A_{2}\left\|u_{\boldsymbol{\alpha}}-v_{h}\right\|_{V}+\sup _{\substack{w_{h} \in V_{h, p} \\ w_{h} \neq 0}} \frac{\sum_{\substack{\boldsymbol{\beta} \in \mathcal{I}_{M, n} \\ 0<\boldsymbol{\beta} \leq \boldsymbol{\alpha}}}\left\langle\mathrm{A}_{\boldsymbol{\beta}}\left(u_{\boldsymbol{\alpha}-\boldsymbol{\beta}}-\hat{u}_{\boldsymbol{\alpha}-\boldsymbol{\beta}}\right), w_{h}\right\rangle \mid}{\left\|w_{h}\right\|_{V}} .
$$

Combining the above inequality with the triangle inequality

$$
\left\|u_{\boldsymbol{\alpha}}-\hat{u}_{\boldsymbol{\alpha}}\right\|_{V} \leq\left\|u_{\boldsymbol{\alpha}}-v_{h}\right\|_{V}+\left\|\hat{u}_{\boldsymbol{\alpha}}-v_{h}\right\|_{V}
$$

we find

$$
\begin{aligned}
& \left\|u_{\boldsymbol{\alpha}}-\hat{u}_{\boldsymbol{\alpha}}\right\|_{V} \leq\left(1+\frac{A_{2}}{C_{1}}\right) \inf _{v_{h} \in V_{h, p}}\left\|u_{\boldsymbol{\alpha}}-v_{h}\right\|_{V} \\
& +\frac{1}{C_{1}} \sup _{\substack{w_{h} \in V_{h, p} \\
w_{h} \neq 0}} \frac{\left|\sum_{\substack{\boldsymbol{\beta} \in \mathcal{I}_{M, n} \\
0<\boldsymbol{\beta} \leq \boldsymbol{\alpha}}}\left\langle\mathrm{A}_{\boldsymbol{\beta}}\left(u_{\boldsymbol{\alpha}-\boldsymbol{\beta}}-\hat{u}_{\boldsymbol{\alpha}-\boldsymbol{\beta}}\right), w_{h}\right\rangle\right|}{\left\|w_{h}\right\|_{V}} .
\end{aligned}
$$


By Assumptions 4.1(c,d),

$$
\left\langle\mathrm{A}_{\boldsymbol{\beta}} u, v\right\rangle \leq C_{\boldsymbol{\beta}}\|u\|_{V}\|v\|_{V}
$$

where $C_{\boldsymbol{\beta}}=C_{\boldsymbol{\beta}}^{\sigma}\left(C_{\boldsymbol{\beta}}^{L}+C_{\boldsymbol{\beta}}^{w}\right)$. Therefore,

$$
\left\|u_{\boldsymbol{\alpha}}-\hat{u}_{\boldsymbol{\alpha}}\right\|_{V} \leq C \inf _{v_{h} \in V_{h, p}}\left\|u_{\boldsymbol{\alpha}}-v_{h}\right\|_{V}+\sum_{\substack{\boldsymbol{\beta} \in \mathcal{I}_{M, n} \\ 0<\boldsymbol{\beta} \leq \boldsymbol{\alpha}}} \hat{C}_{\boldsymbol{\beta}}\left\|u_{\boldsymbol{\alpha}-\boldsymbol{\beta}}-\hat{u}_{\boldsymbol{\alpha}-\boldsymbol{\beta}}\right\|_{V}
$$

for some constants $C$ and $\hat{C}_{\boldsymbol{\beta}}$. The first term on the right-hand side is the approximation error of finite element approximation and the second term is the error propagated from the approximation of lower-order coefficients. Using equation (4.23) recursively, we obtain

$$
\left\|u_{\boldsymbol{\alpha}}-\hat{u}_{\boldsymbol{\alpha}}\right\|_{V} \leq \sum_{\boldsymbol{\beta} \leq \boldsymbol{\alpha}} \hat{C}_{\boldsymbol{\alpha}, \boldsymbol{\beta}} \inf _{v_{h} \in V_{h, p}}\left\|u_{\boldsymbol{\beta}}-v_{h}\right\|_{V}
$$

with some constants $\hat{C}_{\boldsymbol{\alpha}, \boldsymbol{\beta}}$. According to the theory of the finite element approximation $[1,5]$, there exists a positive number $C_{f}$ such that, for every function $u \in H_{0}^{1}(\mathcal{O}) \cap H^{m+1}(\mathcal{O})$,

$$
\inf _{v_{h} \in V_{h}}\left\|u_{\alpha}-v_{h}\right\|_{V} \leq C_{f} h^{\mu-1} p^{-m}\left\|u_{\alpha}\right\|_{H^{m+1}(D)}
$$

where $\mu=\min (m+1, p+1)$. Then

$$
\begin{aligned}
\left\|u_{\boldsymbol{\alpha}}-\hat{u}_{\boldsymbol{\alpha}}\right\|_{V}^{2} & \leq C_{f}^{2} h^{2 \mu-2} p^{-2 m}\left(\sum_{\boldsymbol{\beta} \leq \boldsymbol{\alpha}} \hat{C}_{\boldsymbol{\alpha}, \boldsymbol{\beta}}\left\|u_{\boldsymbol{\alpha}}\right\|_{H^{m+1}(\mathcal{O})}\right)^{2} \\
& \leq C_{f}^{2} h^{2 \mu-2} p^{-2 m} \sum_{\boldsymbol{\beta} \leq \boldsymbol{\alpha}} \hat{C}_{\boldsymbol{\alpha}, \boldsymbol{\beta}}^{2} \sum_{\boldsymbol{\beta} \leq \boldsymbol{\alpha}}\left\|u_{\boldsymbol{\beta}}\right\|_{H^{m+1}(\mathcal{O})}^{2} \\
& =h^{2 \mu-2} p^{-2 m} \hat{C}_{\boldsymbol{\alpha}} \sum_{\boldsymbol{\beta} \leq \boldsymbol{\alpha}}\left\|u_{\boldsymbol{\beta}}\right\|_{H^{m+1}(\mathcal{O})}^{2},
\end{aligned}
$$

and the term $I_{1}$ can be bounded as

$$
\begin{aligned}
I_{1} & \leq h^{2 \mu-2} p^{-2 m} \sum_{\boldsymbol{\alpha} \in \mathcal{J}_{M, n}} \hat{C}_{\boldsymbol{\alpha}} \sum_{\boldsymbol{\beta} \leq \boldsymbol{\alpha}}\left\|u_{\boldsymbol{\beta}}\right\|_{H^{m+1}(D)}^{2}\left\|\mathrm{H}_{\boldsymbol{\alpha}}\right\|_{\mathcal{R} L_{2}(\mathbb{F})}^{2} \\
& \leq h^{2 \mu-2} p^{-2 m} C(M, n) \sum_{\boldsymbol{\alpha} \in \mathcal{J}_{M, n}} \hat{C}_{\boldsymbol{\alpha}}\left\|u_{\boldsymbol{\alpha}}\right\|_{H^{m+1}(D)}^{2}\left\|\mathrm{H}_{\boldsymbol{\alpha}}\right\|_{\mathcal{R} L_{2}(\mathbb{F})}^{2} \\
& \leq h^{2 \mu-2} p^{-2 m} C(M, n) \max _{\boldsymbol{\alpha} \in \mathcal{J}_{M, n}} \hat{C}_{\boldsymbol{\alpha}} \sum_{\boldsymbol{\alpha} \in \mathcal{J}_{M, n}}\left\|u_{\boldsymbol{\alpha}}\right\|_{H^{m+1}(D)}^{2}\left\|\mathrm{H}_{\boldsymbol{\alpha}}\right\|_{\mathcal{R} L_{2}(\mathbb{F})}^{2} \\
& \leq h^{2 \mu-2} p^{-2 m} C_{1}(M, n)\|u\|_{\mathcal{R} L_{2}\left(\mathbb{F} ; H^{m+1}(D)\right)}^{2} .
\end{aligned}
$$

To estimate the truncation error $I_{2}$ of the Wiener chaos expansion, note that $\left\|\mathrm{H}_{\boldsymbol{\alpha}}\right\|_{\mathcal{R} L_{2}(\mathbb{F})}^{2}=r_{\boldsymbol{\alpha}} \boldsymbol{\alpha} !$, and, by (3.8),

$$
r_{\boldsymbol{\alpha}}=\frac{(2 \mathbb{N})^{-2 \alpha} q^{-\alpha}}{\alpha !}
$$

Also,

$$
\left\|u_{\boldsymbol{\alpha}}\right\|_{V} \leq q^{\boldsymbol{\alpha}}\left\|u_{(\mathbf{0})}\right\|_{V} \leq C_{A} q^{\boldsymbol{\alpha}}\|f\|_{H^{-1}(D)} .
$$


As a result,

$$
I_{2}=\sum_{\boldsymbol{\alpha} \in \mathcal{J} \backslash \mathcal{J}_{M, n}}\left\|u_{\boldsymbol{\alpha}}\right\|_{V}^{2}\left\|\mathrm{H}_{\boldsymbol{\alpha}}\right\|_{\mathcal{R} L_{2}(\mathbb{F})}^{2} \leq C_{A}\|f\|_{H^{-1}(D)} \sum_{\boldsymbol{\alpha} \in \mathcal{J} \backslash \mathcal{J}_{M, n}}(2 \mathbb{N})^{-2 \boldsymbol{\alpha}} \leq C_{2}\left(\frac{1}{2^{2 M}}+\frac{1}{n}\right)
$$

where the last inequality is taken from [4], proof of Theorem 2. This, together with (4.17) and (4.26), completes the proof of Theorem 4.4 .

\section{Computations}

In this section we present some results of numerical experiments and compare our model with another one studied in the literature on uncertainty quantification. We consider the following one-dimensional problem

$$
\left\{\begin{array}{l}
-\frac{\mathrm{d}}{\mathrm{d} x}\left(K * \frac{\mathrm{d}}{\mathrm{d} x} u\right)=1 \\
u(0)=0, \quad u(1)=0
\end{array}\right.
$$

where

- * denotes either the Wick product $\diamond$ or the ordinary product · ;

- $K=\mathrm{e}^{c \xi-\frac{1}{2} c^{2}}, \xi$ is a standard Gaussian random variable, $c>0$ is a real number.

The random variable $K$ can be regarded as a simplified version of the random field $\mathrm{e}^{\diamond G(x)}$ from (4.10). Note that

$$
\mathbb{E}[K]=1, \quad \operatorname{Var}[K]=\mathrm{e}^{c^{2}}-1 .
$$

Denote by $\overline{\mathrm{H}}_{i}$ the normalized Hermite polynomials:

$$
\overline{\mathrm{H}}_{i}(\xi)=\frac{\mathrm{H}_{i}(\xi)}{\sqrt{i !}} .
$$

Substituting the truncated Wiener chaos expansion

$$
u(x)=\sum_{i=0}^{n} u_{i}(x) \overline{\mathrm{H}}_{i}(\xi)
$$

into equation (5.1) and implementing the finite element projection we obtain the uncertainty propagators

$$
\begin{gathered}
-\sum_{i=0}^{n} \frac{\mathrm{d}^{2}}{\mathrm{~d} x^{2}} u_{i}(x) \mathbb{E}\left[K \overline{\mathrm{H}}_{i} \overline{\mathrm{H}}_{j}\right]=\delta_{0, j}, \quad j=0,1, \ldots, n \\
-\sum_{i=0}^{n} \frac{\mathrm{d}^{2}}{\mathrm{~d} x^{2}} u_{i}(x) \mathbb{E}\left[\left(K \diamond \overline{\mathrm{H}}_{i}\right) \overline{\mathrm{H}}_{j}\right]=\delta_{0, j}, \quad j=0,1, \ldots, n
\end{gathered}
$$

for the ordinary product and the Wick product, respectively.

Using the Taylor expansion and the properties of Hermite polynomials, we obtain

$$
\begin{gathered}
\mathbb{E}\left[K \overline{\mathrm{H}}_{i} \overline{\mathrm{H}}_{j}\right]=\mathrm{e}^{-c^{2} / 2} \sum_{n=0}^{\infty} \sum_{k=0}^{[n / 2]} \sum_{p \leq i \wedge j} A(n, k) B(i, j, p) \delta_{n-2 k, i+j-2 p}, \\
\mathbb{E}\left[\left(K \diamond \overline{\mathrm{H}}_{i}\right) \overline{\mathrm{H}}_{j}\right]=\frac{\mathrm{e}^{-c^{2} / 2}}{\sqrt{i !}} \sum_{n=0}^{\infty} \sum_{k=0}^{[n / 2]} c^{n} \frac{\sqrt{(n-2 k+i) !}}{(n-2 k) !(2 k) ! !} \delta_{n-2 k+i, j},
\end{gathered}
$$


where

$$
A(n, k)=\frac{c^{n}}{n !}\left(\begin{array}{c}
n \\
2 k
\end{array}\right)(2 k-1) ! ! \sqrt{(n-2 k) !}, \quad B(i, j, p)=\left[\left(\begin{array}{c}
i \\
p
\end{array}\right)\left(\begin{array}{l}
j \\
p
\end{array}\right)\left(\begin{array}{c}
i+j-2 p \\
i-p
\end{array}\right)\right]^{1 / 2} .
$$

Formulas similar to (5.4a) and (5.4b) can also be derived when

$$
K(x)=\mathrm{e}^{\diamond G(x)},
$$

with $G(x)$ given by (4.7). For simplicity, we present numerical results for the space-independent case $G(x)=c \xi$, corresponding to $K=\mathrm{e}^{c \xi-\left(c^{2} / 2\right)}$. The qualitative behavior of the solutions of (5.1), with either ordinary or Wick product, remains the same for the more general noise (5.5), as long as conditions (4.8) hold.

Let us compare the matrix structures of $\mathbb{E}\left[K \overline{\mathrm{H}}_{i} \overline{\mathrm{H}}_{j}\right]$ and $\mathbb{E}\left[\left(K \diamond \overline{\mathrm{H}}_{i}\right) \overline{\mathrm{H}}_{j}\right]$.

- Ordinary product. For all $c_{1}, \ldots, c_{n} \in \mathbb{R}$, we have

$$
\begin{aligned}
\sum_{i, j=1}^{n} c_{i} c_{j} \mathbb{E}\left[K \overline{\mathrm{H}}_{i} \overline{\mathrm{H}}_{j}\right] & =\mathbb{E}\left[\sum_{i, j=1}^{n} c_{i} c_{j} K \overline{\mathrm{H}}_{i} \overline{\mathrm{H}}_{j}\right] \\
& =\mathbb{E}\left[\left(\sum_{i=1}^{n} K^{1 / 2} \overline{\mathrm{H}}_{i} c_{i}\right)^{2}\right] \geq 0 .
\end{aligned}
$$

It can be shown that when $K$ is lognormal, $c_{i}$ must be zero if the equality is satisfied. In other words, the matrix $\mathbb{E}\left[K \overline{\mathrm{H}}_{i} \overline{\mathrm{H}}_{j}\right]$ is symmetric and positive definite. Thus all the eigenvalues of the matrix $\left(\mathbb{E}\left[K \overline{\mathrm{H}}_{i} \overline{\mathrm{H}}_{j}\right], i, j=1, \ldots, n\right)$ are positive, and the system (5.3a) is well-posed. However, all unknowns in equation (5.3a) are coupled together, which is not a desirable property for numerical computation.

- Wick product. As $n$ must be even for $i=j$ to get nonzero terms in equation (5.4b), it follows that $\mathbb{E}\left[\left(K \diamond \overline{\mathrm{H}}_{i}\right) \overline{\mathrm{H}}_{i}\right]=1$ for all $i=0,1, \ldots$ Indeed,

$$
\begin{aligned}
\mathbb{E}\left[\left(K \diamond \overline{\mathrm{H}}_{i}\right) \overline{\mathrm{H}}_{i}\right] & =\frac{\mathrm{e}^{-1 / 2}}{\sqrt{i !}} \sum_{m=0}^{\infty} \sum_{k=0}^{m} \frac{\sqrt{(2 m-2 k+i) !}}{(2 m-2 k) !(2 k) ! !} \delta_{2 m-2 k+i, i} \\
& =\frac{\mathrm{e}^{-1 / 2}}{\sqrt{i !}} \sum_{m=0}^{\infty} \frac{\sqrt{i !}}{(2 m) ! !} \\
& =\mathrm{e}^{-1 / 2} \sum_{m=0}^{\infty} \frac{1}{2^{m} m !}=\mathrm{e}^{-1 / 2} \mathrm{e}^{1 / 2}=1 .
\end{aligned}
$$

Furthermore, we note that $\mathbb{E}\left[\left(K \diamond \overline{\mathrm{H}}_{i}\right) \overline{\mathrm{H}}_{j}\right]$ is a lower-triangular matrix. In other words, the coefficient $u_{i}$ depends only on $u_{j}$ with $j<i$.

In the first numerical experiment, we take $c=1$ and compare the chaos coefficients of solutions corresponding to the ordinary and Wick products. Figure 1 presents the results. For a better comparison, we use the same scale on both graphs and also plot the function $U_{d}$, the solution of

$$
-\frac{\mathrm{d}^{2} U_{d}(x)}{\mathrm{d} x^{2}}=1,0<x<1 ; U_{d}(0)=U_{d}(1)=0 .
$$

Note that $U_{d}$ is the solution of (5.1) when $K$ is replaced by its mean value $\mathbb{E}[K]=1$.

We make the following observations:

(1) When the ordinary product is used, $U_{d} \neq u_{0}$; when the Wick product is used, $U_{d}=u_{0}$. This is a direct consequence of equations (5.3a) and (5.3b). In Figure 2, we plot the function $u_{0}$ corresponding 

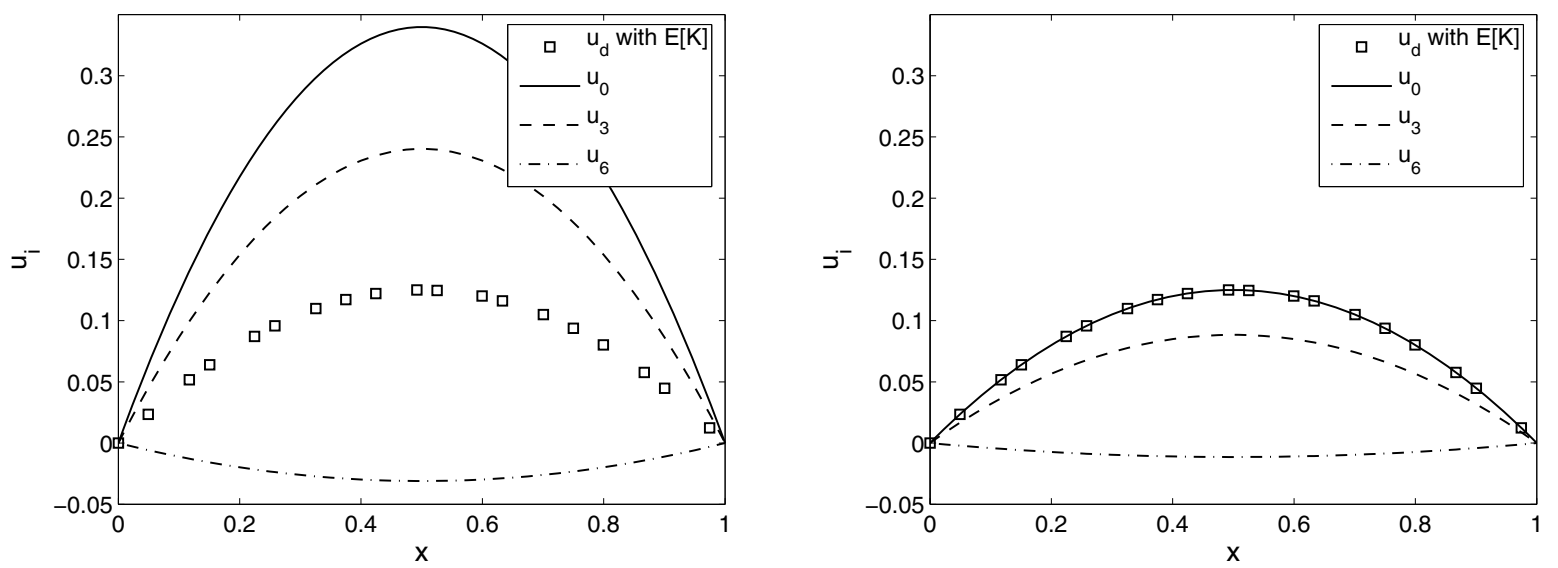

Figure 1. Chaos expansion coefficients. Left: ordinary product; right: Wick product.

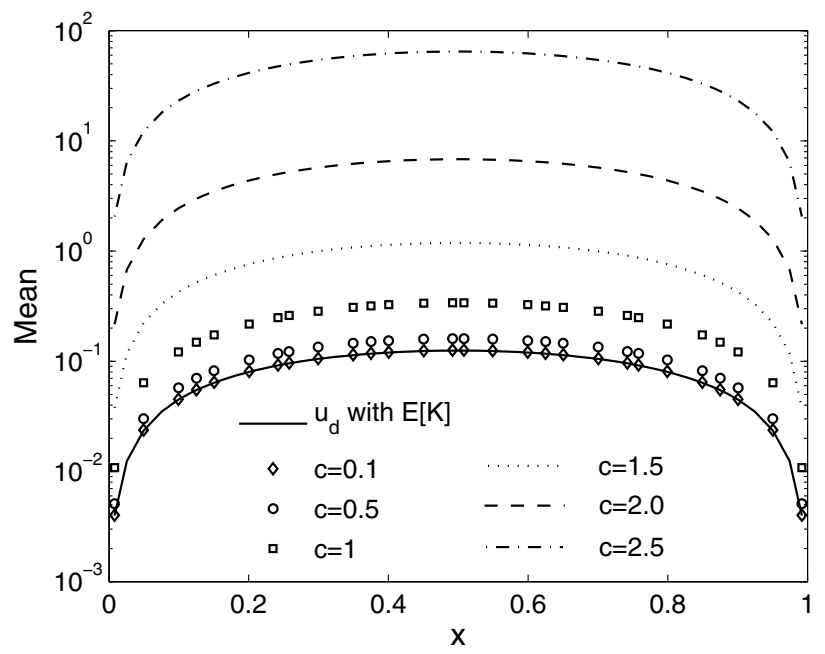

Figure 2. The plot of $\mathbb{E}[u(x)]$ for different values of $c$, ordinary product.

to the ordinary product, for different values of $c$. It appears that $u_{0}$ is an increasing function of $c$ and is close to $U_{d}$ when $c \leq 0.1$.

(2) The larger the number $i$, the smaller the coefficient $u_{i}$. However, it appears that the coefficients for the Wick product are much smaller than the corresponding coefficients for the ordinary product. Below, we investigate this phenomenon further by changing the value of $c$.

In the second numerical experiment, we study the relation between the variance of the perturbation $K$ and the variance of the corresponding solution. We change the value of $c$ from 0.1 to 2.5 , which, by (5.2), is equivalent to changing the standard deviation of $K$ from 0.1003 to 22.7379 . Figures 3 and 4 present the results.

In Figure 3, we plot the variance of the solution as a function of $x$ for several values of $c$, and notice that, in each case, the variance seems to be largest near the middle of the interval $(0,1)$. In Figure 4 , we plot the solution variance at point $x=0.5$ as a function of $c$. It appears that variances corresponding to ordinary and Wick products are close for small $c$, but diverge quickly as $c$ increases: in the same range of the values of $c$, 

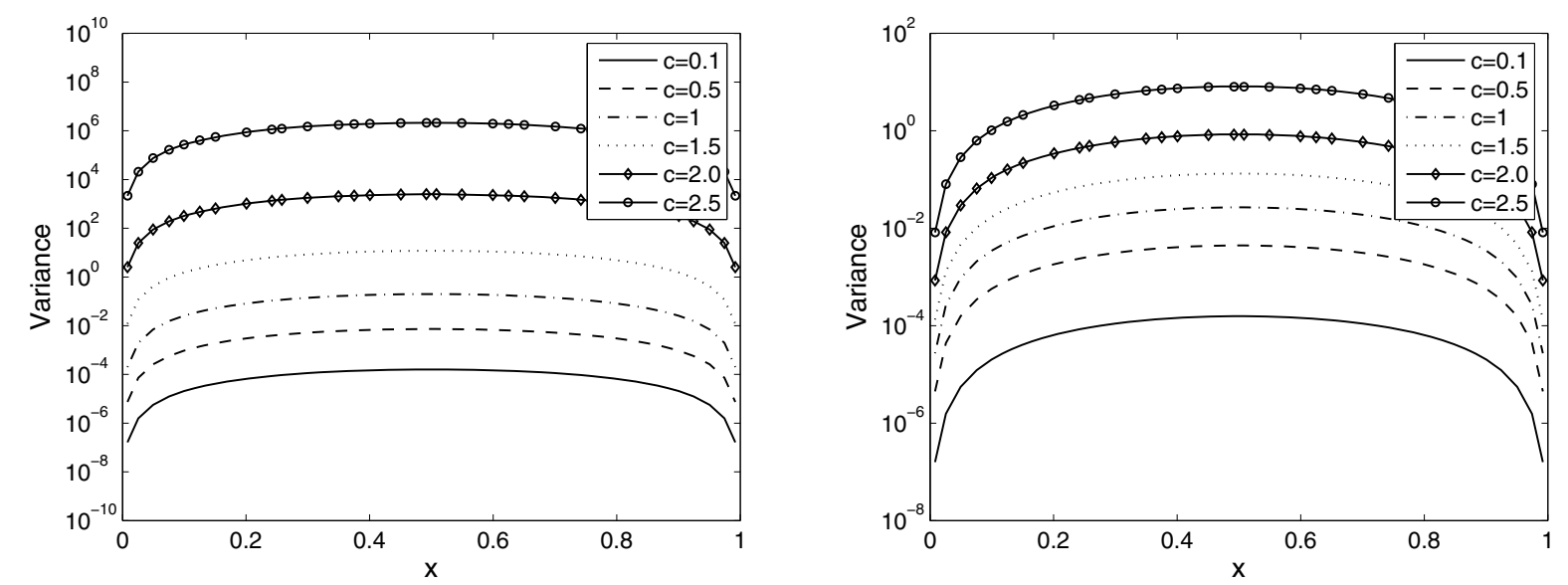

FiguRE 3. Variance of the solution for different values of $c$. Left: ordinary product; right: Wick product.

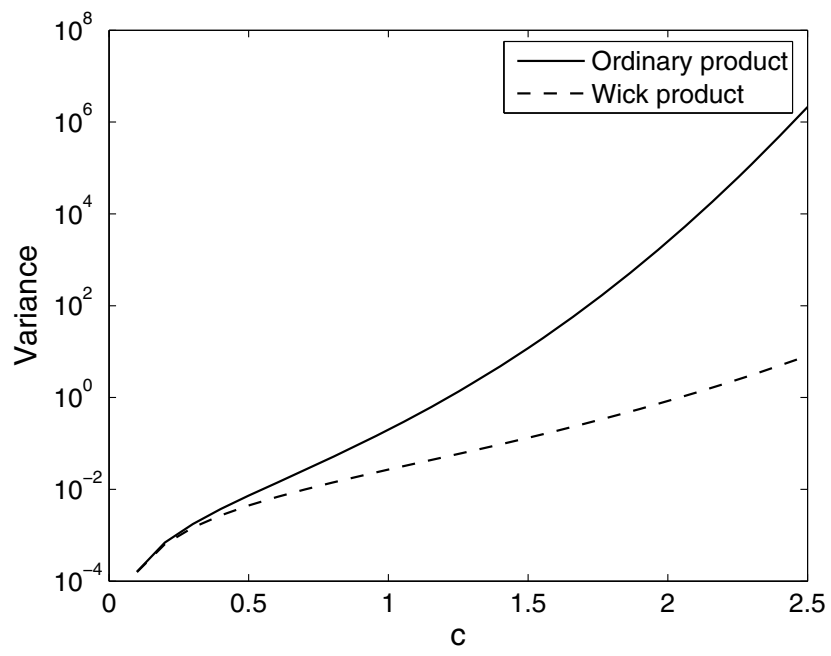

Figure 4. Variance of the solution at $x=0.5$, as a function of $c$.

the variance of the solution increases from $O\left(10^{-5}\right)$ to $O\left(10^{6}\right)$ for the ordinary product, and from $O\left(10^{-5}\right)$ to $O\left(10^{1}\right)$ for the Wick product.

In summary, although the solutions of equation (5.1) with Wick and ordinary products belong to $L_{2}\left(\mathbb{F} ; H_{0}^{1}(0,1)\right)$, the two different products lead to many differences in the properties of the solution. The differences are especially pronounced when the noise $K$ is large. A more detailed analysis shows that many numerical characteristics of the solutions differ by the factor of $\mathrm{e}^{c^{2}}$, which is related to the equality $\mathrm{e}^{c \xi-\frac{1}{2} c^{2}} \diamond \mathrm{e}^{-c \xi-\frac{1}{2} c^{2}}=1$. At this point, we make the statement intentionally vague, because this scaling by the factor $\mathrm{e}^{c^{2}}$ seems to have many important ramifications and must be studied further, both analytically and numerically.

Acknowledgements. S.V. Lototsky acknowledges support from NSF Grant DMS-0803378. B.L. Rozovskii acknowledges support from NSF Grant DMS-0604863, ARO Grant W911NF-07-1-0044, and AFOSR Grant No. FA9550-09-1-0613 (inter). X. Wan acknowledges support from DOE grant SC0002324. 


\section{REFERENCES}

[1] I. Babuška and M. Suri, The $p$ and $h-p$ versions of the finite element method, basic principles and properties. SIAM Rev. 36 (1994) 578-632.

[2] I. Babuška, R. Tempone and G.E. Zouraris, Galerkin finite element approximations of stochastic elliptic partial differential equations. SIAM J. Numer. Anal. 42 (2004) 800-825.

[3] R.H. Cameron and W.T. Martin, The orthogonal development of nonlinear functionals in a series of Fourier-Hermite functions. Ann. Math. 48 (1947) 385-392.

[4] Y. Cao, On convergence rate of Wiener-Ito expansion for generalized random variables. Stochastics 78 (2006) 179-187.

[5] P.G. Ciarlet, The finite element method for elliptic problems, Classics in Applied Mathematics 40. Society for Industrial and Applied Mathematics (SIAM), Philadelphia (2002).

[6] F.W. Elliott, Jr., D.J. Horntrop and A.J. Majda, A Fourier-wavelet Monte Carlo method for fractal random fields. J. Comput. Phys. 132 (1997) 384-408.

[7] T. Hida, H.-H. Kuo, J. Potthoff and L. Sreit, White noise. Kluwer Academic Publishers, Boston (1993).

[8] H. Holden, B. Øksendal, J. Ubøe and T. Zhang, Stochastic partial differential equations. Birkhäuser, Boston (1996).

[9] K. Itô, Stochastic integral. Proc. Imp. Acad. Tokyo 20 (1944) 519-524.

[10] G.E. Karniadakis and S.J. Sherwin, Spectral/hp element methods for computational fluid dynamics. Second edition, Numerical Mathematics and Scientific Computation, Oxford University Press, New York (2005).

[11] Yu.G. Kondratiev, P. Leukert, J. Potthoff, L. Streit and W. Westerkamp, Generalized functionals in Gaussian spaces: the characterization theorem revisited. J. Funct. Anal. 141 (1996) 301-318.

[12] H.-H. Kuo, White noise distribution theory. Probability and Stochastics Series, CRC Press, Boca Raton (1996).

[13] M. Loève, Probability theory - I, Graduate Texts in Mathematics 45. Fourth edition, Springer-Verlag, New York (1977).

[14] S.V. Lototsky and B.L. Rozovskii, Stochastic differential equations driven by purely spatial noise. SIAM J. Math. Anal. 41 (2009) 1295-1322.

[15] D. Nualart, The Malliavin calculus and related topics. Second edition, Probability and its Applications (New York), SpringerVerlag, Berlin (2006).

[16] S. Pilipović and D. Seleši, Expansion theorems for generalized random processes, Wick products and applications to stochastic differential equations. Infin. Dimens. Anal. Quantum Probab. Relat. Top. 10 (2007) 79-110.

[17] S. Pilipović and D. Seleši, On the generalized stochastic Dirichlet problem. Part I: The stochastic weak maximum principle. Potential Anal. 32 (2010) 363-387.

[18] Ch. Schwab, $p$ - and hp-finite element methods, Theory and applications in solid and fluid mechanics. Numerical Mathematics and Scientific Computation, Oxford University Press, New York (1998).

[19] M. Shinozuka and G. Deodatis, Simulation of stochastic processes by spectral representation. AMR 44 (1991) 191-204.

[20] T.G. Theting, Solving Wick-stochastic boundary value problems using a finite element method. Stochastics Stochastics Rep. 70 (2000) 241-270.

[21] G. Våge, Variational methods for PDEs applied to stochastic partial differential equations. Math. Scand. 82 (1998) 113-137.

[22] X. Wan, B. Rozovskii and G.E. Karniadakis, A stochastic modeling methodology based on weighted Wiener chaos and Malliavin calculus. Proc. Natl. Acad. Sci. USA 106 (2009) 14189-14194. 